\title{
Sub-resolution limit spatio-spectral information using Differential Speckle Interferometry
}

\author{
Lagarde, S., Sánchez, L. J., and Petrov, R. G. \\ Département d'Astrophysique, Universit de Nice-Sophia Antipolis, \\ U.R.A. 709 du C.N.R.S, O6108 Nice Cedex 2, France
}

\begin{abstract}
Differential Speckle Interferometry (DSI) combines high angular resolution techniques with medium to high resolution spectroscopy. For non resolved sources, it yields the displacement $\vec{\epsilon}(\lambda)$ of the object photocenter with wavelength. Combined with the spectrum $s(\lambda), \vec{\epsilon}(\lambda)$ can give information with spatial and/or spectral resolution well beyond the telescope or spectrograph limits. This complementarity is illustrated here with experimental results. For the binary system Capella, we measure the angular separation, separate the spectra of the components and derive the radial velocity difference and the rotation velocity of each component. For the slowly rotating star Aldebaran we obtain the position angle of the stellar rotation axis and a relation between the angular diameter and the rotation velocity which in this case gives the latest.
\end{abstract}

\section{Introduction}

Differential speckle interferometry (DSI) is a technique based on the differences existing between the speckle patterns produced by the same source in various spectral channels. When applied to objects much smaller than the telescope Airy disk (Beckers 1982) it yields the angular variation $\vec{\epsilon}(\lambda)$ of the source photocenter with wavelength (Petrov 1989). If $o(\vec{r}, \lambda)$ is the object brightness distribution at wavelength $\lambda, \vec{\epsilon}(\lambda)$ is defined by:

$$
\vec{\epsilon}(\lambda)=\frac{\int \vec{r} o(\vec{r}, \lambda) \overrightarrow{d r}}{\int o(\vec{r}, \lambda) \overrightarrow{d r}}=\frac{d(\lambda)}{s(\lambda)},
$$

where the spectrum $s(\lambda)$ is the zero order moment of $o(\vec{r}, \lambda)$ and $d(\lambda)$ is its first order moment.

In this paper we wish to underline the similarity and complementarity of $\vec{\epsilon}(\lambda)$ and $s(\lambda)$ with two experimental results on the double star Capella (separation $\rho=50.6$ milliarcseconds - mas- and radial velocity difference $\Delta V_{r}=$ $42.3 \mathrm{~km} / \mathrm{s}$ ) and on the slowly rotating star Aldebaran (angular diameter $\Phi=$ $20.5 \mathrm{mas}$ and $V \operatorname{sini} \leq 8 \mathrm{~km} / \mathrm{s}$ ) which we fairly well resolved with the $152 \mathrm{~cm}$ telescope (Airy disk $\simeq 80$ mas) in spite of the poor spectroscopic quality of the instrument (spectral resolution $\simeq 17 \mathrm{~km} / \mathrm{s}$ ). The experimental device was based on a transformation of an existing spetrograph (Petrov et al 1991) and was of the $x-\lambda$ type: at the exit plane of the spectrograph a $2 \mathrm{D}$ photon counting detec- 

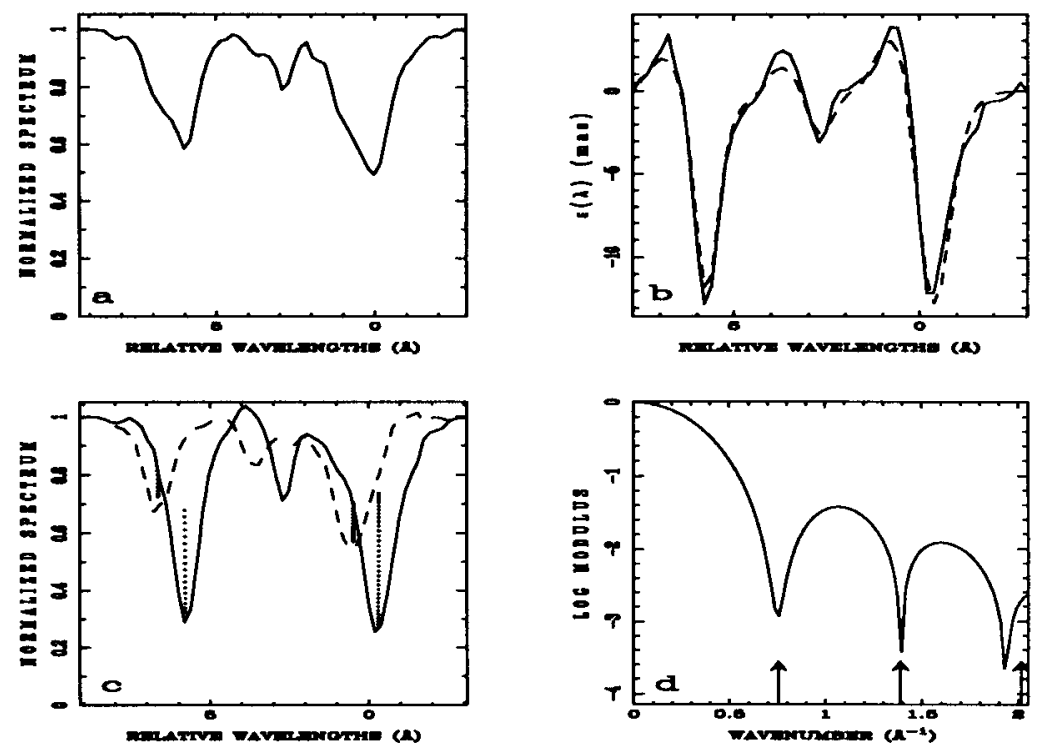

Figure 1. DSI observations of the binary star Capella in the $\mathrm{Na}$ lines. a: normalized spectrum (the wavelengths are measured relatively to the D2 line of the "Sun" component). b: displacement in the separation direction. The uncertainty on the displacement is of the order of 0.5 mas rms. The interrupted line represents the best model of the displacement from the known ephemerides of the star and an estimation of the components spectra. c: spectra of the "Procyon" component (dashed line) and of the "Sun" component (full line) separated by combining the curves in figures a and $b$. d: Modulus of the Fourier transform of the D2 line of the "Procyon" component in a logarithmic scale. The zeros (arrows) correspond to a rotation velocity of $41 \mathrm{~km} / \mathrm{s}$.

tor records a dispersed image of the speckles fed into its slit whose width was reduced to two Airy disks.

\section{DSI observations of the double star Capella}

Figure 1a shows the spectrum obtained from Capella on October 301988 near the Sodium doublet with a very partial resolution of the spectroscopic binarity. The direction of the $\vec{\epsilon}(\lambda)$ displacement gave the binary position angle with a $2^{\circ}$ accuracy and figure $1 \mathrm{~b}$ represents the displacement $\epsilon(\lambda)$ in the separation direction together with a model based on the known ephemerides of Capella (Barlow et al 1993) and assuming that the spectra of its components are correctly modeled by a Solar spectrum affected by a Vsini $=7 \mathrm{~km} / \mathrm{s}$ and a Procyon spectrum with Vsini $=36 \mathrm{~km} / \mathrm{s}$ (Griffin 1986). The uncertainty on the measured $\epsilon(\lambda)$ was of the order of 0.5 mas and was dominated by detector distortion. If we have a good knowledge of the spectroscopic characteristics of Capella, figure 
1b would yield the separation $\rho$ with a $3 \%$ accuracy. On the other hand, if $\rho$ is known, thanks for example to interferometric observations, the combination of $\epsilon(\lambda) / \rho$, which is very easily shown to be equal to the difference between the two spectra, and of $s(\lambda)$ which is their sum, permits to separate the spectra of the components without any further modelling. Figure $1 \mathrm{c}$ shows the result of such an operation, which has a lot of potential applications, ranging from a determination of $\Delta V_{r}$ with a spectral super-resolution, to a much better analysis of the physical properties of each component. As an illustration, figure 1d shows a Fourier analysis of the lines of the fast rotator ("Procyon" component) which permits to measure its $\mathrm{Vsini}=41 \pm 1 \mathrm{~km} / \mathrm{s}$. Recently, observations made at the russian $6 \mathrm{~m}$ telecope with a slightly higher spectral resolution $(\sim 12 \mathrm{~km} / \mathrm{s})$ gave for the "Solar" component Vsini $=8.5 \pm 1 \mathrm{~km} / \mathrm{s}$. It is also possible to use this spectrum separation for an iterative determination of $\rho$ based on criteria of spectroscopic likeliness. For example if we select the separation which gives us individual lines with bissectors close to the ones in the Sun and Procyon spectroscopic atlases (dots in figure 1c we get $\rho=51 \pm 1$ mas (Lagarde 1994).

\section{DSI observations of the slowly rotating star Aldebaran}

The amplitude of the expected photodisplacement on Aldebaran being of the order of 1 mas, we observed it in the $5560-5600 \AA$ region where there are a lot of deep lines, as it can be seen in figure 2a. Figure 2b shows the measured $\epsilon(\lambda)$ together with a model deduced from the measured spectrum with a Vsini assumed equal to $6 \mathrm{~km} / \mathrm{s}$.

The photodisplacement is maximum perpendicularly to the rotation axis and figure $2 \mathrm{c}$ shows the least-squares fitted value of the measure to model ratio $\alpha$ as it varies with the angle $\beta$ between the axis on which $\vec{\epsilon}(\lambda)$ is projected and the south-north axis. The position angle of the star, defined as the angle between the N-S and the rotation axes was found to be $\theta=110 \pm 5^{\circ}$. The error on $\theta$ results from the SNR on $\alpha$, which reached 10 after combination of all usable spectral channels (the figures $2 a$ and $2 b$ show less than one half of the actually used spectral range). This capacity to measure stellar position angles, unique to DSI, can be applied to the study of the formation and evolution of multiple stellar systems or of the relations between stellar rotation and circumstellar envelopes. It has been shown (Lagarde 1994) that when the Vsini of a star is substancially smaller than the FWHM of the local line profile $p(\lambda)$ (resulting from the convolution of the Voigt profile by the spectral point spread function), the combination of $\vec{\epsilon}(\lambda)$ and $s(\lambda)$ can yield only the product $\Phi$ Vsini independently from $p(\lambda)$. The procedure is as follows. An hypothesis for Vsini together with the measured $s(\lambda)$ yields $p(\lambda)$. Then $p(\lambda)$, Vsini and $s(\lambda)$ are used to produce a model of $\vec{\epsilon}(\lambda)$ which differs from the average measure only by a proportionality factor which is the stellar diameter $\Phi$. Repeating this operation produces the function $\Phi=f($ Vsini) represented in figure 2d. Again, known Vsini, would give a measure of $\Phi$ with a $10 \%$ accuracy. In the case of a slow rotating giant like Aldebaran, which has been very often resolved by long baseline interferometry, the known angular diameter $(\Phi=20.5 \pm 0.5$ mas, $D i$ Benedetto 1993) yields an unusual measure of $\mathrm{V} \operatorname{sini}=5.8 \pm 1 \mathrm{~km} / \mathrm{s}$ made even more remarkable by the $17 \mathrm{~km} / \mathrm{s}$ point spread function and the $\sim 10 \mathrm{~km} / \mathrm{s}$ typical Aldebaran's Voigt pro- 

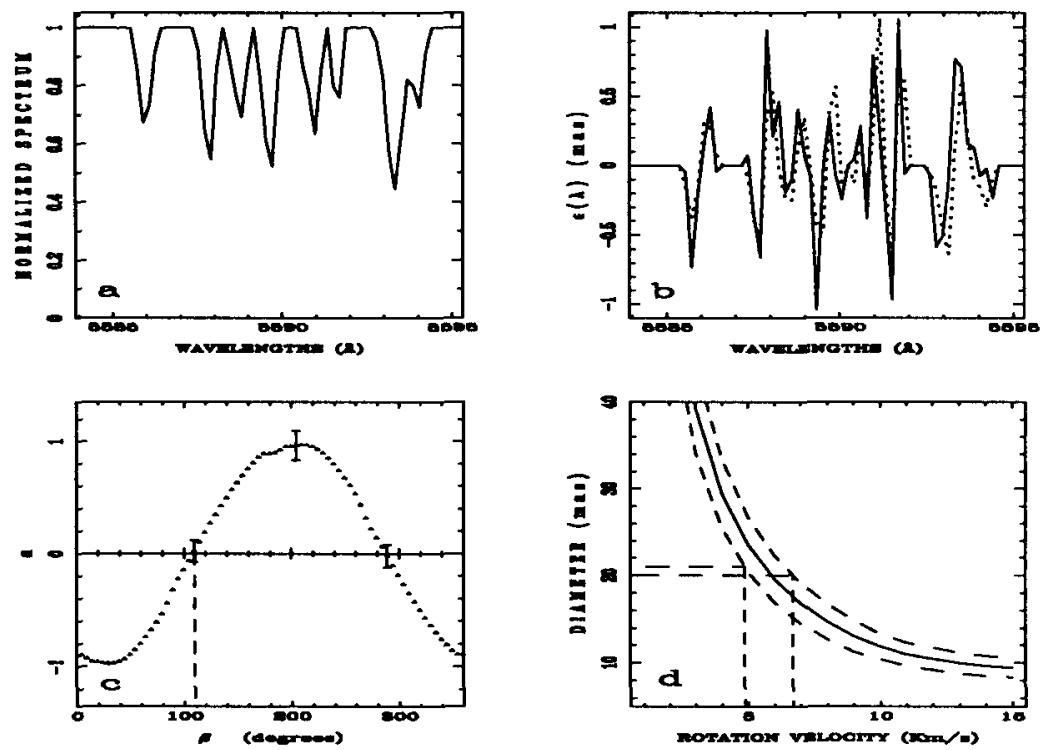

Figure 2. DSI observations of Aldebaran. a: spectrum near $5590 \AA$. b: measured photodisplacement (full line) and model based on $\Phi=$ $20.5 \mathrm{mas}$ and Vsini $=6 \mathrm{~km} / \mathrm{s}$ (dashed line). The uncertainty on $\vec{\epsilon}(\lambda)$ was of the order of 0.6 mas. c: ratio $\alpha$ between the measure and the model as a function of the angle $\beta$ between the axis on which $\vec{\epsilon}(\lambda)$ is projected and the south-north axis. The position angle $\theta$ of the stellar rotation axis is $110 \pm 5^{\circ}$. d: relation between the angular diameter $\Phi$ and V sini deduced from the combination of $s(\lambda)$ and $\epsilon(\lambda)$. The full line represents $\Phi$ and the two interrupted lines stand for $\Phi \pm \sigma_{\Phi}$. The known value of $\Phi(20.5 \pm 0.5$ mas $)$ yields $\mathrm{Vsini}=5.8 \pm 1 \mathrm{~km} / \mathrm{s}$.

file (FWHM). Then we can say that for spectroscopically resolved rotators, DSI will yield stellar diameters of bright stars with the resolution of an hectometric baseline interferometer, while for interferometrically resolved slow rotators, it will yield rotation velocities at levels unreachable up to know.

\section{Conclusion}

We have illustrated the links between DSI and spectroscopy with results on sources which were well beyond the spatial and spectral resolution limits. For objects with well described spectra and velocity fields, DSI will yield unambiguous spatial information while for sources with known angular structure it will give spectroscopic and velocity data well beyond the classical limits. In the intermediate cases, DSI will provide spatio-spectral relations which will constrain the models. We are convinced that this very general property will be found again for most of the very numerous DSI applications such as the study of the density-velocity relations in circumstellar envelopes or in AGNs, and, when DSI 
is implemented on the VLTI, asteroseismology or Doppler imaging of spotted or magnetic stars.

\section{Acknowledgements}

The observations were made at the "Observatoire de Haute Provence" operated by the CNRS and we used the photon counting intensified CCD "CP 40" developed and kindly operated by A. Blazit and R. Foy.

\section{Discussion}

T. Herbst: Can you give us an idea of the physical size of the instrument you described?

R.G. Petrov: A speckle camera with a Courtès bichromator and resolution up to 5000 will fit in a tube less than $1 \mathrm{~m}$ long and $80 \mathrm{~cm}$ in diameter. Our $\mathrm{x}-\lambda$ experiment gives us a 70000 maximum resolution and fits into a tube $140 \mathrm{~cm}$ long and $70 \mathrm{~cm}$ in diameter, thanks to a two mirrors camera with equivalent focal length $\sim 4.5 \mathrm{~m}$. This relative compacity is permitted because there is no use for a slit more than 2 or 3 speckles wide. A detailed description of our last experiment can be found in "Cuevas, S. Ph.D. Thesis, Orsay University, December 17, 1993".

\section{References}

Barlow, D. J. Fekel, F. C. \& Scarfe, C. D. 1993, PASP, 105, 476

Beckers, J. M. 1982, Opt. Acta, 29, 361

Di Benedetto, G. P. 1993, A\&A, 270, 315

Griffin, R. \& R. 1986, J. Astrophys. Astr. 7, 45

Lagarde, S.:1994, Traitement des données et premiers résultats en interférométrie différentielle des tavelures, $\mathrm{Ph}$. D. Thesis, University of Nice.

Petrov, R. G.: 1989, in Proc. NATO ASI on Diffraction limited imaging with very large telescopes, Cargese, Corse. Eds. J. M. Mariotti and D. M. Alloin, 249

Petrov, R. G. Balega, Y. Y. Blazit, A. Borgnino, J. Foy, R. Lagarde, S. Martin, F. \& Vassyuk V. V.: 1991, in Proc. ESO Conf. on High Resolution Imaging by Interferometry II, Garching, Germany. Eds. J. M. Beckers and F. Merkle, 435 